\section{Opioidanalgetika bei Gonarthrose: häufigere Einnahme bei Begleiterkrankungen}

King LK et al. Are medical comorbidities contributing to the use of opioid analgesics in patients with knee osteoarthritis? Osteoarthritis and Cartilage 2020; 28: 1030-1037

Obwohl Opioidanalgetika im Allgemeinen nicht zur Behandlung einer Gonarthrose empfohlen werden, kommen sie dennoch häufig zum Einsatz. Eine kanadische Arbeitsgruppe hat den Zusammenhang zwischen Begleiterkrankungen und der von Gonarthrose-Patienten berichteten Verwendung von Opioidanalgetika untersucht.

Für ihre Querschnittsstudie rekrutierten die Autorinnen und Autoren Patientinnen und Patienten, zweier Hüft- und Kniekliniken in Alberta, Kanada, die für eine Knieendoprothetik überwiesen wurden. Mittels standardisierter Fragebögen wurden demografische Merkmale wie Alter, Geschlecht, Einkommen, Bildung, soziale Unterstützung und Raucherstatus erfasst. Mittels WOMACScore (Western Ontario and McMaster Universities Osteoarthritis Index) wurden die Stärke der Schmerzen, Steifigkeit oder Behinderung bestimmt und mit dem KOOSScore Schmerzen, Behinderung und Funktion erfasst. Ebenfalls dokumentiert wurden die jemals durchgeführten und aktuellen, nicht-chirurgischen Therapien der Gonarthrose, und schließlich die Begleiterkankungen, die aus einer Liste mit ja oder nein angegeben wurden.

Mittels multivariabler Poisson-Regression, mit einer robusten Schätzung der Standardfehler, wurde der Zusammenhang zwischen Begleiterkrankungen und dem aktuellen Opioidkonsum, unter Beachtung möglicher Störfaktoren, bewertet.

Insgesamt gaben 2127 Patientinnen und Patienten mit Gonarthrose einen aktuellen Opioidkonsum an und wurden in die Analyse eingeschlossen. Ihr Durchschnittsalter betrug $65,4 \pm 9,1$ Jahre, 59,2\% waren weiblich und $56,5 \%$ hatten eine weitere Ausbildung nach einem allgemeinbildenden Schulabschlusses oder eines beruflichen Abschlusses absolviert. 91,2\% hatten Übergewicht oder Adipositas (BMI $>25 \mathrm{~kg} / \mathrm{m}^{2}$ ) und 77,9\% hatten mindestens eine zusätzliche koexistierende Erkrankung. Am häufigsten waren Hypertonie mit 50,6\%, Rückenschmerzen mit 50,5\%, Depression mit 22,0\%, Diabetes mit 16,7\% und Herzerkrankungen mit 12,1\%. Die Schmerzdurchschnittswerte nach dem WOMAC-Score betrugen 58,2 $\pm 18,0$ Punkte (von 100) und die Körperfunktion gemäß KOOS 56,4 \$17,5 Punkte (von 100). Die Anzahl betroffener Gelenke betrug im Mittel 2.

Die aktuelle Behandlung der Gonarthrose war in 57,6\% der Fälle Bewegung und/oder Physiotherapie, in 61,1 \% der Fälle eine medikamentöse Behandlung mit NSAIDs und in 29,8\% der Fälle eine Therapie mit OpioidAnalgetika.

In der multivariablen Regressionsanalyse, unter Einbeziehung potenzieller Störfaktoren, waren begleitende Hypertonie, Magen-Darm-Erkrankungen, depressive Verstimmung und eine höhere Anzahl problematischer Gelenke mit dem Opioidkonsum assoziiert. Keinen Zusammenhang mit dem Opioidkonsum wurde mit der jemals empfohlenen opioidfreien, pharmakologischen Behandlung oder einer nicht-pharmakologischen Therapie festgestellt. Das Risiko des aktuellen Opioidkonsums erhöhte sich in einer Dosis-Wirkungs-Beziehung mit zunehmender Zahl an Begleiterkrankungen.

\section{FAZIT}

In einer großen Kohorte von Patientinnen und Patienten mit Gonarthrose und 12 überprüften Begleiterkrankungen, waren Hypertonie, Magen-Darm-Erkrankungen und depressive Verstimmungen mit der aktuellen Anwendung von Opioid-Analgetika assoziiert und zwar zusätzlich zur Gesamtbelastung durch belastete Gelenke. Nach Meinung des Autorenteams sind verbesserte Leitlinien zur Behandlung einer Arthrose mit häufig vergesellschafteten Begleiterkrankungen erforderlich.

Richard Kessing, Zeiskam 